\title{
Successful Treatment of Adult-Onset Erythromelalgia with Steroid Pulse and Pregabalin
}

\author{
Aya Kakizaki Taku Fujimura Yumi Kambayashi \\ Akiko Watabe Setsuya Aiba \\ Department of Dermatology, Tohoku University Graduate School of Medicine, \\ Sendai, Japan
}

\section{Key Words}

Erythromelalgia $\cdot$ Steroid pulse $\cdot$ Pregabalin

\begin{abstract}
Adult-onset erythromelalgia (EM) is a rare disease characterized by episodic bouts of burning pain and erythema for which the optimal therapy is unclear. In this report, we describe a 68year-old Japanese woman with adult-onset EM. Intravenous administration of methylprednisolone sodium succinate $1,000 \mathrm{mg} /$ day dramatically improved her pain as evaluated by the visual analog scale. Although the patient's pain gradually developed again, it could be controlled with pregabalin. Our present case might suggest a possible, optimal therapy for adult-onset EM.
\end{abstract}

\section{Introduction}

Erythromelalgia (EM) is a rare disease characterized by painful swelling and erythema on the extremities [1-3]. The symptoms are often triggered by minor trauma, exercise, increased skin temperature, or heat exposure and often alleviated by cooling and elevation of the involved extremity. There are three distinct types: primary EM, secondary EM and EM with thrombocythemia [4]. Primary EM is observed most commonly in patients aged less than 30 years without underlying disorders. Secondary $\mathrm{EM}$ is associated with several autoimmune disorders [e.g. systemic lupus erythematosus (SLE), insulin-dependent diabetes mellitus]. The optimal therapy for the first two types of EM is still under discussion. In contrast, EM with thrombocythemia is usually treated with aspirin, since the burning pain is relatively easy to control [4]. In 
this report, we describe a case of adult-onset EM successfully controlled with steroid pulse and pregabalin.

\section{Case Report}

A 68-year-old Japanese woman presented at our outpatient clinic with a 10-year history of painful, recurrent erythema on her bilateral hands. There was no erythema on her feet. She had been diagnosed with idiopathic EM and treated with oral intake of vitamin E $150 \mathrm{mg} /$ day, transamin capsules $750 \mathrm{mg} /$ day, prostaglandin E1 $15 \mu \mathrm{g} /$ day and aspirin $100 \mathrm{mg} /$ day, without any improvement. On her initial visit, physical examination revealed dark erythematous swelling on her bilateral hands ( fig. 1a). In addition, she complained of heavy pain and said she could hardly sleep. The full blood count test was within the normal range. The blood picture was normal, which denied myeloproliferative disorders or hematological malignancy. The biochemical profile revealed normal levels of antinuclear antibodies $(\times 40)$ and a slightly decreased IgG level $(702 \mathrm{mg} / \mathrm{dl})$. The serum Creactive protein was negative. The hemoglobin A1c level was 5.3\%. No specific autoantibodies (e.g. dsDNA, ssDNA, SS-A, SS-B) were detected. Prothrombin time, activated partial thromboplastin time, serum fibrinogen, and platelet count were within normal ranges. There was no evidence of liver and kidney dysfunction. There was no evidence of abnormal electrocardiographic pattern. The patient was found to be euthyroid by a measurement of the basal TSH and free T4 levels as well as by TRH provocative test. Internal malignancy was excluded at the internal department. From the above findings, we diagnosed the patient as having adult-onset EM of unknown origin. We administered methylprednisolone sodium succinate $1,000 \mathrm{mg} /$ day intravenously for 3 days, and after that we treated her with oral prednisolone 60,40 and $20 \mathrm{mg} /$ day for 2 days each. Two days after the administration of methylprednisolone sodium succinate, eruption and pain had improved (fig. 1b). The visual analog scale (VAS) score improved from 10 to 2 (fig. 2a). However, 6 days after therapy start, her pain gradually developed again (VAS score 2 to 5 ). Then, we administered pregabalin 150 $\mathrm{mg} /$ day. The VAS score improved again, and the patient has been able to control her pain for half a year, although slight erythema could still be observed.

\section{Discussion}

EM is a rare condition characterized by episodic bouts of burning pain, erythema, and edema, most commonly involving the extremities [1]. Symptoms are often triggered by minor trauma, exercise, or heat exposure and often alleviated by cooling and elevation of the involved extremity. Several reports have suggested successful treatments for EM [2-5], but the optimal therapy is still under discussion. In this report, we describe a case of adult-onset EM successfully controlled with steroid pulse and pregabalin as evaluated by VAS.

Previous reports suggested an association between secondary forms of EM and autoimmune disorders $[3,6,7]$. Indeed, there is evidence that secondary EM might be associated with an autoimmune condition in which there are autoantibodies directed against the sodium channel encoded for by the SCN9A gene [3]. In addition, Jackson and Oates [3] reported a case of autoimmune-related EM; however, these autoantibodies were not suggested to be directly associated with EM like SLE. On the contrary, the primary form of EM is associated with a mutation in the sodium channel Nav1.7, which is selectively expressed within the dorsal root ganglion and sympathetic ganglia and encoded by the SCN9A gene $[3,8]$.

Once diagnosis is established, potential secondary causes must be excluded [6, 9]. A complete blood count is recommended for screening and follow-up. Other causes associated with EM include SLE, Raynaud's disease, pernicious anemia, thrombotic 
thrombocytopenic purpura, infectious mononucleosis, metabolic, endocrine, and vascular origin and diabetic neuropathy $[6,7,9]$. Appropriate testing should be considered based on diagnostic suspicion of the above conditions. Although there was no evidence for autoimmune-associated EM in our present case, late onset of EM might indicate the possible involvement of autoantibodies. In addition, Suh et al. [5] reported that adult-onset idiopathic EM could be successfully treated with corticosteroids and pentazocine. Furthermore, Pfund et al. [9] reported successful treatment of EM with high-dose methylprednisolone. For the above reasons, we decided to administer steroid pulse and pregabalin, and monitored the patient's pain with VAS. VAS is a simple assessment tool consisting of a $10-\mathrm{cm}$ line with 0 on one end, representing no pain, and 10 on the other end, representing the worst pain ever experienced, which a patient marks to indicate the severity of his or her pain (fig. 2b). In our present case, the VAS score and erythema on the hands immediately improved after administration of high-dose methylprednisolone sodium succinate and were maintained by oral pregabalin. Although we describe a single case and the observation is limited, our case might suggest a possible, optimal therapy for adult-onset EM. Further study is needed to confirm this limited observation.

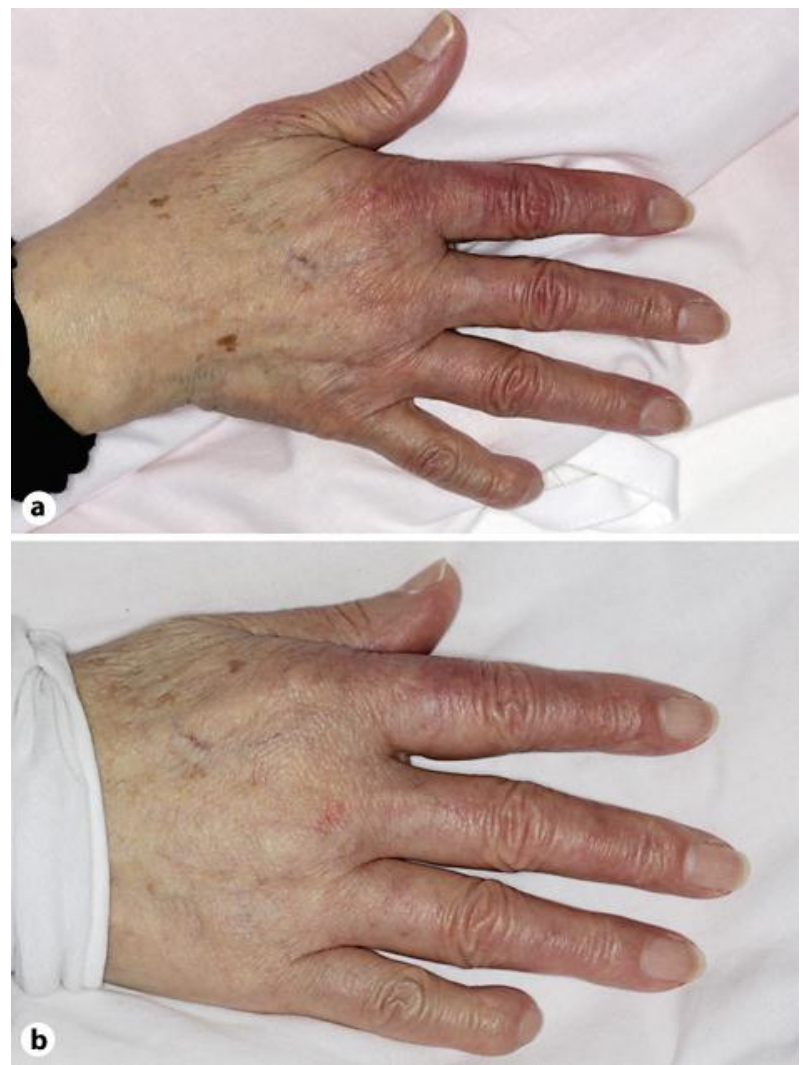

Fig. 1. Dark erythematous swelling on the patient's right hand. a Day 0: before treatment. b Day 3: after treatment. 


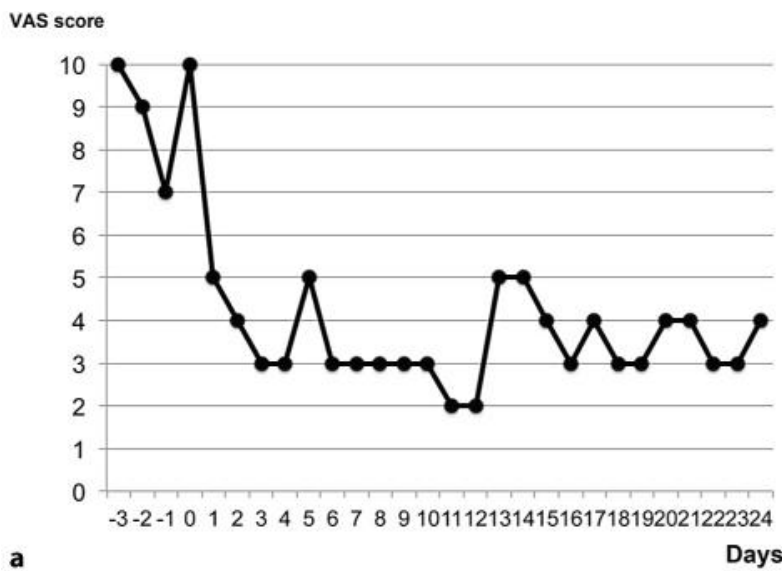

\section{Visual Analogue Scale}

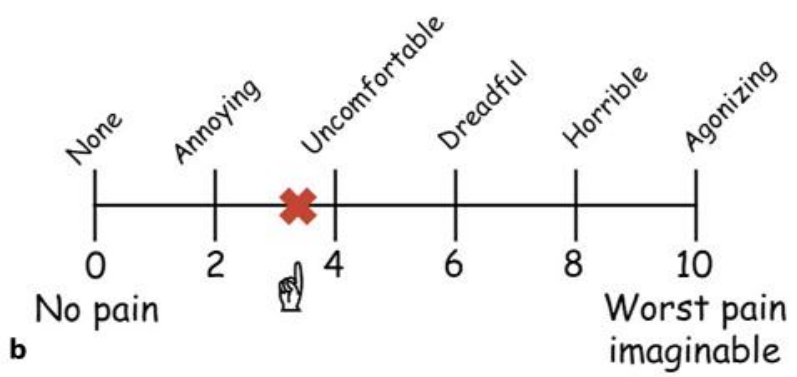

Fig. 2. a Monitoring for VAS before and after the administration of methylprednisolone sodium succinate. Day 0: start of administration of methylprednisolone sodium succinate. Day 8: start of oral intake of pregabalin. Day 13: accidental trauma (the patient hit her hand). b VAS score: assessment tool consisting of a $10-\mathrm{cm}$ line, which the patient marks to indicate her pain.

\section{References}

1 Waxman SG, Dib-Hajj S: Erythermalgia: molecular basis for an inherited pain syndrome. Trends Mol Med 2005;11:555-562.

-2 Moody S, Pacheco S, Butler IJ, Koenig MK: Secondary erythromelalgia successfully treated with intravenous immunoglobulin. J Child Neurol 2012;27:922-923.

3 Jackson AL, Oates JA: A patient with adult erythermalgia: evidence suggesting an autoimmune etiology. Am J Med Sci 2008;335:320-322.

4 Seishima M, Kanoh H, Izumi T, Niwa M, Matsuzaki Y, Takasu A, Ban M, Kitajima Y: A refractory case of secondary erythromelalgia successfully treated with lumbar sympathetic ganglion block. Br J Dermatol 2000;143:868-872

5 Suh DH, Kim SD, Ahn JS, Han KH, Cho KH, Eun HC, Youn JI: A case of erythromelalgia successfully controlled by systemic steroids and pentazocine - is it related to a unique subtype of neutrophilic dermatosis? J Dermatol 2000;27:204-210.

6 Gaur S, Koroscil T: Late-onset erythromelalgia in a previously healthy young woman: a case report and review of the literature. J Med Case Rep 2009;3:106.

7 Badeloe S, Henquet CJ, Nieuwhof CM, Frank J: Secondary erythromelalgia involving the ears probably preceding lupus erythematosus. Int J Dermatol 2007;46(suppl 3):6-8. 
-8 Yang Y, Wang Y, Li S, Xu Z, Li H, Ma L, Fan J, Bu D, Liu B, Fan Z, Wu G, Jin J, Ding B, Zhu X, Shen Y: Mutations in SCN9A, encoding a sodium channel alpha subunit, in patients with primary erythermalgia. J Med Genet 2004;41:171-174.

9 Pfund Z, Stankovics J, Decsi T, Illes Z: Childhood steroid-responsive acute erythromelalgia with axonal neuropathy of large myelinated fibers: a dysimmune neuropathy? Neuromuscul Disord 2009;19:49-52. 